\title{
Charged Wormholes in Spacetimes of Embedding Class One
}

\author{
Peter K. F. Kuhfittig \\ Department of Mathematics, Milwaukee School of Engineering, Milwaukee, Wisconsin 53202-3109, USA
}

\begin{abstract}
The existence of charged black holes has suggested that wormholes may also be charged. The purpose of this paper is to construct a general model of a charged wormhole that proves to be a natural extension of the original Morris-Thorne wormhole. This goal is achieved by means of the classical embedding theory that has played a major role in the general theory of relativity.
\end{abstract}

Keywords: charged wormholes, embedding class one DOI: 10.31526/LHEP.2021.207

\section{INTRODUCTION}

Wormholes are handles or tunnels in spacetime connecting widely separated regions of our Universe or different universes in a multiverse. Apart from some forerunners, macroscopic traversable wormholes were first proposed by Morris and Thorne [1]. The wormhole geometry is described by the following static and spherically symmetric line element

$$
d s^{2}=-e^{v(r)} d t^{2}+\frac{d r^{2}}{1-\frac{b(r)}{r}}+r^{2}\left(d \theta^{2}+\sin ^{2} \theta d \phi^{2}\right),
$$

using units in which $c=G=1$. Here $v=v(r)$ is called the redshift function, which must be everywhere finite to prevent the appearance of an event horizon. The function $b=b(r)$ is commonly referred to the shape function since it determines the spatial shape of the wormhole when viewed, for example, in an embedding diagram [1]. The spherical surface $r=r_{0}$ is called the throat of the wormhole, where $b\left(r_{0}\right)=r_{0}$. The shape function must also meet the requirements $b^{\prime}\left(r_{0}\right)<1$, called the flare-out condition, while $b(r)<r$ for $r>r_{0}$. A final requirement is asymptotic flatness: $\lim _{r \rightarrow \infty} v(r)=0$ and $\lim _{r \rightarrow \infty} b(r) / r=0$.

The flare-out condition can only be met by violating the null energy condition (NEC)

$$
T_{\alpha \beta} k^{\alpha} k^{\beta} \geq 0
$$

for all null vectors $k^{\alpha}$, where $T_{\alpha \beta}$ is the energymomentum tensor. Matter that violates the NEC is called "exotic" in [1] and is usually confined to a narrow region around the throat. For the outgoing null vector $(1,1,0,0)$, the violation of the NEC becomes

$$
T_{\alpha \beta} k^{\alpha} k^{\beta}=\rho+p_{r}<0 .
$$

Here $T_{t}^{t}=-\rho$ is the energy density, $T_{r}^{r}=p_{r}$ is the radial pressure, and $T_{\theta}^{\theta}=T_{\phi}^{\phi}=p_{t}$ is the lateral (transverse) pressure.
In the area of wormhole physics, an interesting extension was proposed by Kim and Lee [2]. Motivated by the Reissner-Nordström spacetime, they considered the following line element for a charged wormhole:

$$
\begin{aligned}
d s^{2}= & -\left(1+\frac{Q^{2}}{r^{2}}\right) d t^{2}+\frac{d r^{2}}{1-\frac{b(r)}{r}+\frac{Q^{2}}{r^{2}}} \\
& +r^{2}\left(d \theta^{2}+\sin ^{2} \theta d \phi^{2}\right),
\end{aligned}
$$

where $Q$ is the electric charge. The purpose of this paper is to extend this special model to a general MorrisThorne wormhole with electric charge by starting with a spacetime of embedding class one, discussed in the next section. By assuming that the redshift function is also dependent on the charge $Q$, a possible line element is

$$
\begin{aligned}
d s^{2}= & -e^{v\left(r, Q^{2}\right)} d t^{2}+\frac{d r^{2}}{1-\frac{b(r)}{r}+\frac{Q^{2}}{r^{2}}} \\
& +r^{2}\left(d \theta^{2}+\sin ^{2} \theta d \phi^{2}\right) .
\end{aligned}
$$

Even though the metric has changed, we would like $b=b(r)$ to retain the usual properties of a shape function in a Morris-Thorne wormhole, although this is not a requirement in [2]. We will see later, however, that due to the embedding, the shape function actually has the form $b=b\left(r, Q^{2}\right)$. The line element will also be modified to produce a model for a charged wormhole that generalizes the original Morris-Thorne wormhole in a natural way.

\section{THE EMBEDDING}

Embedding theorems have played a major role in the general theory of relativity, as exemplified by the induced-matter theory in [3]: if the embedding of spacetime is carried out in accordance with Campbell's theorem, then the resulting five-dimensional theory can explain the origin of matter. Like any mathematical model, the main criterion for its acceptance is its usefulness. In this paper, we are primarily interested in spacetimes of embedding class one, a special case of the more general class $m$ : an $n$-dimensional Riemannian space is 
said to be of embedding class $m$ if $m+n$ is the lowest dimension of the flat space in which the given space can be embedded. The resulting mathematical model has proved to be extremely useful in the study of compact stellar objects $[4,5,6,7,8,9]$ and will be used in this paper to extend the above Kim-Lee model. To that end, we first recall that the exterior Schwarzschild solution is a Riemannian space of embedding class two. Following [4], we will assume that a spherically symmetric metric of class two can be reduced to a metric of class one by a suitable transformation of coordinates. To see how, we start with the spherically symmetric line element

$$
d s^{2}=e^{v(r)} d t^{2}-e^{\lambda(r)} d r^{2}-r^{2}\left(d \theta^{2}+\sin ^{2} \theta d \phi^{2}\right),
$$

where $v$ and $\lambda$ are differentiable functions of the radial coordinate $r$. It is shown in [4] that this metric of class two can be reduced to a metric of class one and can therefore be embedded in the five-dimensional flat spacetime

$$
d s^{2}=-\left(d z^{1}\right)^{2}-\left(d z^{2}\right)^{2}-\left(d z^{3}\right)^{2}-\left(d z^{4}\right)^{2}+\left(d z^{5}\right)^{2} .
$$

This reduction can be accomplished by the following transformation: $z^{1}=r \sin \theta \cos \phi, z^{2}=r \sin \theta \sin \phi$, $z^{3}=r \cos \theta, z^{4}=\sqrt{K} e^{v / 2} \cosh \frac{t}{\sqrt{K}}$, and $z^{5}=$ $\sqrt{K} e^{v / 2} \sinh \frac{t}{\sqrt{K}}$. The differentials of the components are:

$$
\begin{gathered}
d z^{1}=\sin \theta \cos \phi d r+r \cos \theta \cos \phi d \theta-r \sin \theta \sin \phi d \phi \\
d z^{2}=\sin \theta \sin \phi d r+r \cos \theta \sin \phi d \theta+r \sin \theta \cos \phi d \phi \\
d z^{3}=\cos \theta d r-r \sin \theta d \theta \\
d z^{4}=\sqrt{K} e^{v / 2} \frac{v^{\prime}}{2} \cosh \frac{t}{\sqrt{K}} d r+e^{v / 2} \sinh \frac{t}{\sqrt{K}} d t
\end{gathered}
$$

and

$$
d z^{5}=\sqrt{K} e^{v / 2} \frac{v^{\prime}}{2} \sinh \frac{t}{\sqrt{K}} d r+e^{v / 2} \cosh \frac{t}{\sqrt{K}} d t .
$$

To facilitate the substitution into Eq. (7), we first obtain the expressions for $-\left(d z^{1}\right)^{2}-\left(d z^{2}\right)^{2}-\left(d z^{3}\right)^{2}$ and $-\left(d z^{4}\right)^{2}+\left(d z^{5}\right)^{2}:$

$-\left(d z^{1}\right)^{2}-\left(d z^{2}\right)^{2}-\left(d z^{3}\right)^{2}=-d r^{2}-r^{2}\left(d \theta^{2}+\sin ^{2} \theta d \phi^{2}\right)$

and

$$
-\left(d z^{4}\right)^{2}+\left(d z^{5}\right)^{2}=e^{v} d t^{2}-\frac{1}{4} K e^{v}\left(v^{\prime}\right)^{2} d r^{2} .
$$

Substituting Eqs. (13) and (14) in Eq. (7), we obtain the new metric

$$
\begin{aligned}
d s^{2}= & e^{v} d t^{2}-\left[1+\frac{1}{4} K e^{v}\left(v^{\prime}\right)^{2}\right] d r^{2} \\
& -r^{2}\left(d \theta^{2}+\sin ^{2} \theta d \phi^{2}\right)
\end{aligned}
$$

So metric (15) is equivalent to metric (6) if

$$
e^{\lambda}=1+\frac{1}{4} K e^{v}\left(v^{\prime}\right)^{2},
$$

where $K>0$ is a free parameter. Eq. (16) can also be obtained from the Karmarkar condition [10]

$$
R_{1414}=\frac{R_{1212} R_{3434}+R_{1224} R_{1334}}{R_{2323}}, \quad R_{2323} \neq 0,
$$

which is equivalent to the above reduction. In fact, Eq. (16) is a solution to the differential equation

$$
\frac{v^{\prime} \lambda^{\prime}}{1-e^{\lambda}}=v^{\prime} \lambda^{\prime}-2 v^{\prime \prime}-\left(v^{\prime}\right)^{2}
$$

readily solved by separation of variables. So $K$ is actually an integration constant [5].

Next, referring to line element (6), to produce a wormhole solution, we prefer the opposite signature:

$$
d s^{2}=-e^{v(r)} d t^{2}+e^{\lambda(r)} d r^{2}+r^{2}\left(d \theta^{2}+\sin ^{2} \theta d \phi^{2}\right),
$$

which takes us back to line element (5) with $b=$ $b\left(r, Q^{2}\right)$ :

$$
\begin{aligned}
d s^{2}= & -e^{v\left(r, Q^{2}\right)} d t^{2}+\frac{d r^{2}}{1-\frac{b\left(r, Q^{2}\right)}{r}+\frac{Q^{2}}{r^{2}}} \\
& +r^{2}\left(d \theta^{2}+\sin ^{2} \theta d \phi^{2}\right) .
\end{aligned}
$$

As before, we assume that $v\left(r, Q^{2}\right)$ is a differentiable function of $r$ with $\lim _{r \rightarrow \infty} v\left(r, Q^{2}\right)=0$.

\section{THE WORMHOLE SOLUTION}

From Eqs. (16) and (18), we obtain

$$
1-\frac{b\left(r, Q^{2}\right)}{r}+\frac{Q^{2}}{r^{2}}=\frac{1}{1+\frac{1}{4} K e^{v\left(r, Q^{2}\right)}\left[v^{\prime}\left(r, Q^{2}\right)\right]^{2}},
$$

where the prime denotes the derivative with respect to $r$. Solving for $b\left(r, Q^{2}\right)$, we have

$$
b\left(r, Q^{2}\right)=r\left(1+\frac{Q^{2}}{r^{2}}-\frac{1}{1+\frac{1}{4} K e^{v\left(r, Q^{2}\right)}\left[v^{\prime}\left(r, Q^{2}\right)\right]^{2}}\right)
$$

In line element (18), the effective shape function is given by

$$
b_{\mathrm{eff}}\left(r, Q^{2}\right)=b\left(r, Q^{2}\right)-\frac{Q^{2}}{r} .
$$

So, due the embedding,

$$
b_{\mathrm{eff}}\left(r, Q^{2}\right)=r\left(1-\frac{1}{1+\frac{1}{4} K e^{v\left(r, Q^{2}\right)}\left[v^{\prime}\left(r, Q^{2}\right)\right]^{2}}\right) .
$$

Unfortunately, the condition $b_{\text {eff }}\left(r_{0}, Q^{2}\right)=r_{0}$ is now impossible to meet. It quickly becomes apparent, however, that the effective shape function in Eq. (21) implies 
that Eq. (20) needs a slight adjustment:

$$
\begin{aligned}
b\left(r, Q^{2}\right)= & r\left(1+\frac{Q^{2}}{r^{2}}-\frac{1}{1+\frac{1}{4} K e^{v\left(r, Q^{2}\right)}\left[v^{\prime}\left(r, Q^{2}\right)\right]^{2}}\right) \\
& +\frac{Q^{2}}{r} .
\end{aligned}
$$

As noted in Section 1, we would like $b\left(r, Q^{2}\right)$ to satisfy all the properties of a Morris-Thorne wormhole. This goal can be readily achieved thanks to the free parameter $K$ in the embedding theory. In particular, to satisfy the condition $b\left(r_{0}, Q^{2}\right)=r_{0}$, we let

$$
K=\frac{\frac{r_{0}^{2}}{2 Q^{2}}-1}{\frac{1}{4} e^{v\left(r_{0}, Q^{2}\right)}\left[v^{\prime}\left(r_{0}, Q^{2}\right)\right]^{2}}, \quad Q \neq 0 .
$$

Then

$$
\begin{aligned}
& b\left(r, Q^{2}\right)=r\left[1+\frac{Q^{2}}{r^{2}}\right. \\
& \left.-\left(1+\frac{\frac{r_{0}^{2}}{2 Q^{2}}-1}{\frac{1}{4} e^{v\left(r_{0}, Q^{2}\right)}\left[v^{\prime}\left(r_{0}, Q^{2}\right)\right]^{2}} \frac{1}{4} e^{v\left(r, Q^{2}\right)}\left[v^{\prime}\left(r, Q^{2}\right)\right]^{2}\right)^{-1}\right] \\
& +\frac{Q^{2}}{r} .
\end{aligned}
$$

It follows at once that $b\left(r_{0}, Q^{2}\right)=r_{0}$. Using this shape function, we obtain a Morris-Thorne wormhole with a nonzero electric charge.

As noted earlier, asymptotic flatness requires that $\lim _{r \rightarrow \infty} v\left(r, Q^{2}\right)=0$. Since $v\left(r, Q^{2}\right)$ is a differentiable function of $r$, we also have $\lim _{r \rightarrow \infty} v^{\prime}\left(r, Q^{2}\right)=$ 0 . Eq. (25) then yields the other condition, i.e., $\lim _{r \rightarrow \infty} b\left(r, Q^{2}\right) / r=0$.

To check the flare-out condition at or near the throat, we assume that $r \approx r_{0}$ in Eq. (25). Then

$$
b\left(r, Q^{2}\right) \approx r\left(1+\frac{Q^{2}}{r^{2}}-\frac{2 Q^{2}}{r_{0}^{2}}\right)+\frac{Q^{2}}{r}
$$

and

$$
b^{\prime}\left(r_{0}, Q^{2}\right) \approx 1-\frac{4 Q^{2}}{r_{0}^{2}}<1,
$$

provided that $r_{0}>2|Q|, Q \neq 0$.

For the final condition, $b\left(r, Q^{2}\right)<r$ near $r=r_{0}$, we simply let $r_{1} \gtrsim r_{0}$ and observe that

$$
0<\frac{b\left(r_{1}, Q^{2}\right)}{r_{1}} \approx 1+\frac{Q^{2}}{r_{1}^{2}}-\frac{2 Q^{2}}{r_{0}^{2}}+\frac{Q^{2}}{r_{1}^{2}}<1
$$

since $Q \neq 0$.
In summary, a general model for a charged wormhole is given by

$$
d s^{2}=-e^{v\left(r, Q^{2}\right)} d t^{2}+\frac{d r^{2}}{1-\frac{b\left(r, Q^{2}\right)}{r}}+r^{2}\left(d \theta^{2}+\sin ^{2} \theta d \phi^{2}\right),
$$

where $b\left(r, Q^{2}\right)$ is the shape function in Eq. (25). The result is a natural generalization of a Morris-Thorne wormhole.

\section{CONCLUSIONS}

The existence of charged black holes has suggested that wormholes may also be charged. This paper begins with a discussion of Morris-Thorne wormholes, followed by a charged wormhole model due to Kim and Lee [2]. To extend this special model, we made use of the classical embedding theory that is normally viewed as a viable and effective mathematical model. More precisely, we made use of the fact that a spherically symmetric metric of class two can be reduced to a metric of class one by a suitable transformation of coordinates. So the Kim-Lee model is not only extended, the embedding theory yields the following natural generalization of a Morris-Thorne wormhole with electric charge:

$$
d s^{2}=-e^{v\left(r, Q^{2}\right)} d t^{2}+\frac{d r^{2}}{1-\frac{b\left(r, Q^{2}\right)}{r}}+r^{2}\left(d \theta^{2}+\sin ^{2} \theta d \phi^{2}\right),
$$

where

$$
\begin{aligned}
& b\left(r, Q^{2}\right)=r\left[1+\frac{Q^{2}}{r^{2}}\right. \\
& \left.-\left(1+\frac{\frac{r_{0}^{2}}{2 Q^{2}}-1}{\frac{1}{4} e^{v\left(r_{0}, Q^{2}\right)}\left[v^{\prime}\left(r_{0}, Q^{2}\right)\right]^{2}} \frac{1}{4} e^{v\left(r, Q^{2}\right)}\left[v^{\prime}\left(r, Q^{2}\right)\right]^{2}\right)^{-1}\right] \\
& +\frac{Q^{2}}{r}
\end{aligned}
$$

and $r_{0}>2|Q|, Q \neq 0$. Both the redshift and shape functions have the required properties of a MorrisThorne wormhole, while the wormhole spacetime itself is asymptotically flat.

\section{CONFLICTS OF INTEREST}

The author declares that there are no conflicts of interest regarding the publication of this paper.

\section{References}

[1] M. S. Morris and K. S. Thorne, Amer. J. Phys. 56, 395-412 (1988).

[2] S.-W. Kim and H. Lee, Phys. Rev D 63, 064014 (2001). 
[3] P. Wesson, Int. J. Mod. Phys. D 24, 1530001 (2015).

[4] S. K. Maurya and M. Govender, Eur.Phys. J. C 77, 347 (2017).

[5] S. K. Maurya and S. D. Maharaj, Eur. Phys. J. C 77, 328 (2017).

[6] S. K. Maurya, B. S. Ratanpal and M. Govender, Ann. Phys. 382, 36-49 (2017).

[7] S. K. Maurya, Y. K. Gupta, S. Ray and D. Deb, Eur. Phys. J. C 77, 45 (2017).

[8] S. K. Maurya, Y. K. Gupta, S. Ray and D. Deb, Eur. Phys. J. C 76, 693 (2016).

[9] S. K. Maurya, D. Deb, S. Ray and P. K. F. Kuhfittig, Int. J. Mod. Phys. D 28, 1950116 (2019).

[10] K. R. Karmarkar, Proc. Ind. Acad. Sci. 27, 56 (1948). 